\title{
ADAPTIVE BEAMFORMING WITH MVDR ALGORITHM FOR ASSESSMENT ENERGY EFFICIENCY IN CELLULAR NETWORK
}

\author{
Samira ACHKI* \\ Computer Science Department, Faculty of Science and Technique \\ Cadi-Ayyad University, Marrakech, Morocco \\ Yassine JANDI \\ Physics Department, Faculty of Science Semlalia, \\ Cadi Ayyad University Marrakech, Morocco \\ Fatima GHARNATI \\ Physics Department, Faculty of Science Semlalia, \\ Cadi Ayyad University Marrakech, Morocco

\section{Abdellah AIT OUAHMAN} \\ Physics Department, Faculty of Science Semlalia, \\ Cadi Ayyad University Marrakech, Morocco \\ *Corresponding Author Email: S.ichki@gmail.com
}

\begin{abstract}
Base station (BS) and mobile phones. The (BS) equipment and downlink transmission consume the major energy of cellular networks. One of the promising solutions for decrease Energy consumption (EC) and increasing throughput is the deployment of large antenna arrays that can transmit extremely concentrate beams. In the present work, we propose a new antenna muting scheme for beamforming based on Minimum Variance Distortion Less Response MVDR. The operation of MVDR is based on finding the optimum weight to direct the main lobe beam to the desired user location with a unity gain, Implementation results confirmed that the proposed technique is more efficient than the traditional process of the cellular network.
\end{abstract}

Keywords: Cellular Network, Base Station (BS), Energy Efficient (EE), Energy consumption (EC), Beamforming Minimum Variance Distortion Less Response MVDR.

Cite this Article: Samira ACHKI, Yassine JANDI, Fatima GHARNATI, Abdellah AIT OUAHMAN, Adaptive Beamforming with MVDR Algorithm for Assessment Energy Efficiency in Cellular Network. International Journal of Civil Engineering and Technology, 11(2), 2020, pp. 211-219.

https://iaeme.com/Home/issue/IJCIET?Volume=11\&Issue=2 


\section{INTRODUCTION}

Since the number of wireless and cellular networks, as well as the number of mobile users, explodes, energy efficiency was considered a primary major concern in the design and operation of wireless communication systems. Indeed, the energy consumption problem in the Information and Communication Technology (ICT) sector has become critical during the past years. On the one hand, ICT should play a major key role in reducing and minimizing energy consumption in many different sectors such as transportation, power, agriculture, telecommunication, etc....

The telecommunication section and principally cellular networks are important parts of Information and Communication Technology (ICT). With new technologies like Fifth Generation $(5 \mathrm{G})$ and Fourth Generation (4G) coming to the market, this section will grow more in the future. Recently, telecommunication sectors are responsible for about $12 \%$ of the total energy consumption of the world and generate approximately $1 \%$ of $\mathrm{CO} 2$ emissions [1] with percentages expected to rise further. In [2] the authors suggest the deployment of LTE macro base station (BS) to study the impact of modulation and coding schemes (MCS) [3], bandwidth (BW) size and power transmitted on the energy efficiency for an urban environment. They showed that the higher transmission power marks and result in decrease EE. In cellular networks as show in figure 1, the key of energy users firstly is base stations (BSs), backhaul servers and routers. Round $80 \%$ energy is consumed by the BSs and downlink transmission contributes to the major proportion [4]. Because of this statistic, the majority of the energy-saving and reducing research had been a concentrate on the BS. However, the great number of active antenna elements (AAEs), and radio chains necessitate vast energy consumption on the next generation NodeB (eNB).

On the other hand, the most part beamforming techniques have been considered for use at the base station (BS) since antenna arrays are not feasible at mobile terminals due to space limitations.

This paper contains two sections: Section 1 reviewed the works done in studying the energy Efficient in wireless systems communication. Section 2 explains the methodology of our model and algorithm. Section detailed the implementation phase and compares our proposed work.

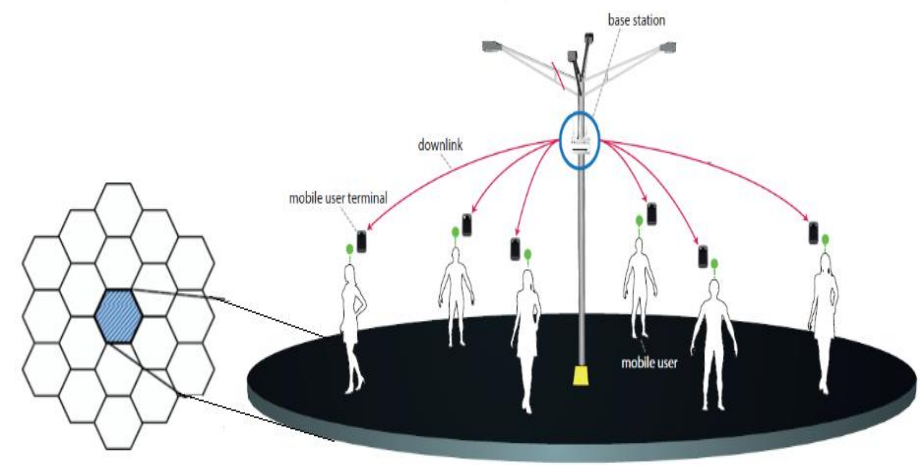

Figure 1 Downlink transmission in cellular network

\section{RELATED WORK}

Optimization of energy consumption in wireless networks was considered a critical need, imposed by the physical constraint which is the lifetime of batteries of embedded equipment such as mobile phones and sensors. 
Several models of energy consumption have been proposed in the literature. These models depend mainly on the type of node. In sensor networks, the radio interface is the component that consumes the bulk of the battery with about $95 \%$ more [4].

Hardware proposal scheme and protocol designs are some big areas of interest of research development in communication systems, which allowed reduction energy consumed by BS and downlink transmission. Despite all inventions, the problem of energy remains a barrier to any technology. Several projects such as Green Radio and EARTH [5], eWin[6], and OPERA-Net[7], confirm that the BS is the most network element, which consumes more energy.

The first project focus on enhancing the energy metrics and models and the energy efficiency hardware and architectures and the management of Energy efficient resource. The second project aims at optimizing the architectures and managing the resource energy. Moreover, it allows the enhancement of the energy metrics and targets and radio technologies and components. The third project improved EE architectures and resource management. The last one considered the link level and technology enablers and the network testbed.

In literature, we have different approaches that resolve the increasing of energy consumption at BS. Among these solutions, the cross-layer optimization [8] and the switching off strategy and beamforming technique [9]. However, they did not take into account the user mobility.

Transmit beamforming can be used to enhance the two conflicting targets for improved EE in multiple-user multiple-input multiple-output (MU-MIMO) communications: mitigating MU interference to maximize the users' information throughput and jamming eavesdroppers to control the leakage of information. However, the current approach to treat both EE and SEE. In [11] is based on costly zero-forcing beamformers, which completely cancel the MU interference and signals received at the eavesdroppers.

For that, we proposed technique Beamforming using the MVDR algorithm. That allows optimizing energy. The Beamforming technique used can save transmission energy to fined the use optimum, will be explained with more details in section three.

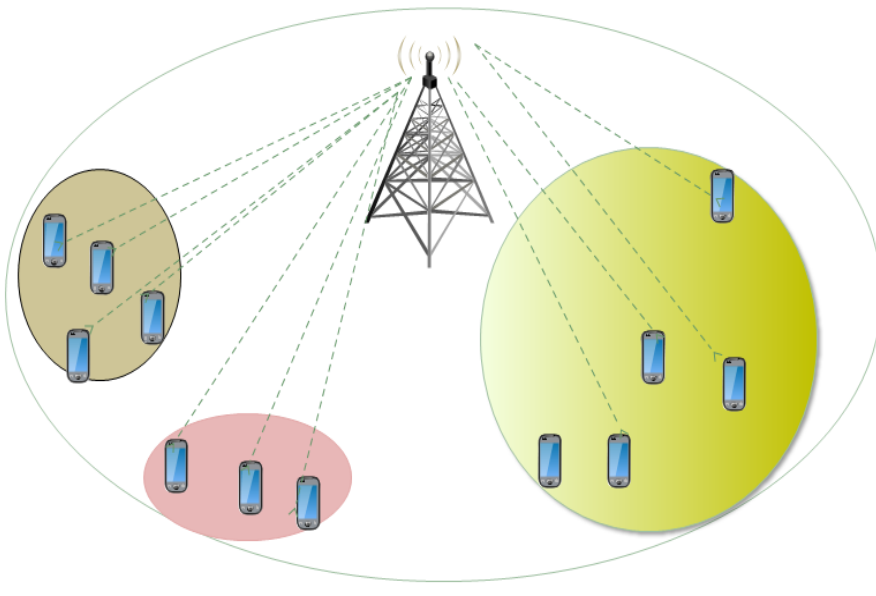

Figure 2 Conceptual model 


\section{SYSTEM MODEL AND ENERGY-EFFICIENT BEAMFORMING SCHEME}

This Section illustrate the system model with one base station and number of user mobile as show in figure 2 , The user mobile UE is randomly positioned in the coverage area of the base station Cell.

\subsection{Data Rate Model}

We consider the downlink in this paper. The data rate for a given user $\mathrm{U}$ located in a sector $\mathrm{s}$ at time slot $t$ is computed according to the Shannon formula [12]:

$$
R_{U, t}=\alpha_{u, t} W \log \left(1+\operatorname{SIN} R_{u, t}\right)
$$

Where $\mathrm{W}$ represents the frequency bandwidth, $\alpha_{u, t}$ the fraction of the bandwidth allocated to user $\mathrm{U}$ in time slot $\mathrm{t}$ and $S I N R_{u, t}$ the SINR of a user $\mathrm{U}$ during a time slot $\mathrm{t}$.

$\alpha(\mathrm{u}, \mathrm{t})$ is determined by the scheduling algorithm used and the configuration of users served by sector $\mathrm{s}$. We concentrate on Round-Robin scheduling thus:

$$
\alpha_{u, t}=\frac{1}{\eta_{s, t}}
$$

Where $\eta_{s, t}$ is the number of users served by sector $\mathrm{s}$ in time slot $\mathrm{t}$.

The SINR is calculated as follows [13]:

$$
S I N R_{u, t}=\frac{p_{s} H_{s, l}(k)}{I_{u, t}+N_{0}}
$$

Where

Ps is the transmit power of sector s.

$H_{s, l}(k)$ is the channel plus antenna gain from sector s to user $\mathrm{k}$ with the antenna of sector $\mathrm{s}$ configured for user $\mathrm{k}$. The antenna configuration generally concerns the beam used.

N0 is the Noise variance.

$\mathrm{Iu}, \mathrm{t}$ is the interference computed as follows:

$$
I_{u, t}=\sum_{s \neq s^{*}} \frac{1}{n_{s *, t}} \sum_{j \in s^{*}} p_{s} H_{s, l}(k)
$$

$P_{u, s, k}$ : is the power consumption of the user $\mathrm{u}$ during period $\mathrm{k}$ in which he was served by the BSs. It takes into account which beam is used by UE so that we can assume exactly his power consumption.

\subsection{Direction of Arrival Estimation}

The Estimate of the Direction of Arrival (DOA) [14] is a technique that applies at the level of the BS, in which the base station, which equipped with a network of antennas instead of a single antenna, performs a scan to detect if there is a user. At the end of scanning, the BS determines the position of the user.

In this part, our objective is to find the direction of the desired user is and the interference in order to steer the main beam towards the desired user and null along the interference. We have calculated the angle of direction using MVDR algorithm. 


\subsection{Beam-Forming}

The beamforming [15] is a technology that allows a network of antenna pointing the outreach to more precise steering, or otherwise, does not radiate not where there is a user. The beamforming can be achieved by several algorithms, we consider a network of antennas formed of a single cell with $\mathrm{M}$ mobile users and base station with L-element antenna array is considered. Let there be d desired signal sources and 1 interference sources transmitting on the same frequency channel simultaneously. Then the signal received by antenna array becomes [16]:

$$
\mathrm{X}(t)=\sum_{j=1}^{d} \mathrm{~A}_{j}(\varphi) S_{j}(t)+\sum_{K=1}^{1} A_{\text {int-k }}(\varphi) S_{k}(t)+N(t)
$$

Where:

Ai $(\varphi)$ is an array steering vector denoting the amplitude gain and phase shift of the signal of $i^{\text {th }}$ user.

$\mathrm{j}$ denotes the desired users and $\mathrm{k}$ denote interferences.

$S_{j}(t)$ is signal vector and $\mathrm{N}(\mathrm{t})$ is random noise component with zero mean and unity variance.

The system output beamformer can be written as:

$$
\mathrm{Z}(t)=\omega^{H} X(t)
$$

where

$\omega$ is weight vector and superscript

$\mathrm{H}$ denote conjugate transpose

\subsection{Algorithm Minimum Variance Distortion Less Response (MVDR)}

Algorithm (Minimum Variance Distortion less Response) MVDR [17] algorithm proposed by Capon en 1967, this algorithm characterizes the type Beamforming algorithm (Beamformer). MVDR beamformer allows minimizes the total output power after beamforming and minimizes interference and noise in the desired direction through adjusting a with factor weight while simultaneously keeping the gain of the array towards the desired signal fixed.

Therefore, any reduction in the output energy obtained by suppressing interference or noise. Mathematically, MVDR weight vector is given as [18]:

$$
\omega=\frac{R^{-1} \mathrm{~A}_{d}(\phi)}{A_{d}(\phi)^{H} R^{-1} A_{d}(\phi)}
$$

Where $\mathrm{R}$ is array correlation matrix defined by

$$
R=E[x(t) x H(t)]
$$

And $\mathrm{E}$ is the vector of the cross-correlation.

\subsection{Power Consumption Model}

The power model of macro BS defined in has linear relationship between average radiated power per and average power consumption; the power consumption can be changed according to the BS and the traffic load level and the BS [19].

$$
P_{\text {con }}=K N_{\text {sect }} N_{a t n}\left(\varsigma P_{f d}+B\right)
$$

Where:

$P_{\text {con }}:$ The total power per BS 
$P_{f d}:$ The power fed to the antenna

$N_{\text {sect }}$ : The BS's number of sectors

$N_{a t n}$ : The number of antennas per sector

$\mathrm{K}$ : The load factor where $\mathrm{K} \epsilon[0,1]$

B: Power that consumed independent of the average power transmits.

\subsection{Energy Consumption Model}

The total energy efficiency EET is defined with of the total amount data, delivered and the total power consumed in bits per joule, is represented by [20]:

$$
E_{E T}=\sum_{i+1}^{N b s} D i / \sum_{i=1}^{N b s} P_{c o n i}
$$

Where

Di : Total data rate with $\mathrm{BSi}$

$P_{\text {coni }}:$ Power consumption

$N_{b s i}$ : Total number of BSs

\section{SIMULATION AND RESULTS ANALYSIS}

We use an LTE event-based simulator. We constructed a system simulator for evaluating and assessing the performance of energy, an event corresponds to arrival or a departure in the whole network. We assume that the users arrive according to a Poisson process, no mobility is considered in the simulations but the users arrive at random locations in the total network figure 3. We concentrate on a dense urban case with an inter-site. Detailed of our system parameters are summarized in table 1.

Table 1 Denotation of the used parameters

\begin{tabular}{|lc|}
\hline \multicolumn{1}{|c|}{ Parameters } & Assumed value \\
\hline Macro Cell layout & Hexagonal trisector \\
Antenna height & $30 \mathrm{~m}$ \\
Bandwidth & $10 \mathrm{MHz}$ \\
Carrier frequency & $2,0 \mathrm{GHz}$ \\
Path loss & $128.1+37.6+\log _{10}(\mathrm{~d}) \mathrm{dB}$ \\
Shadowing & Log-normal $(6 \mathrm{~dB})$ \\
Scheduling type & Round Robin \\
Average file size & 4 Mbits \\
\hline
\end{tabular}




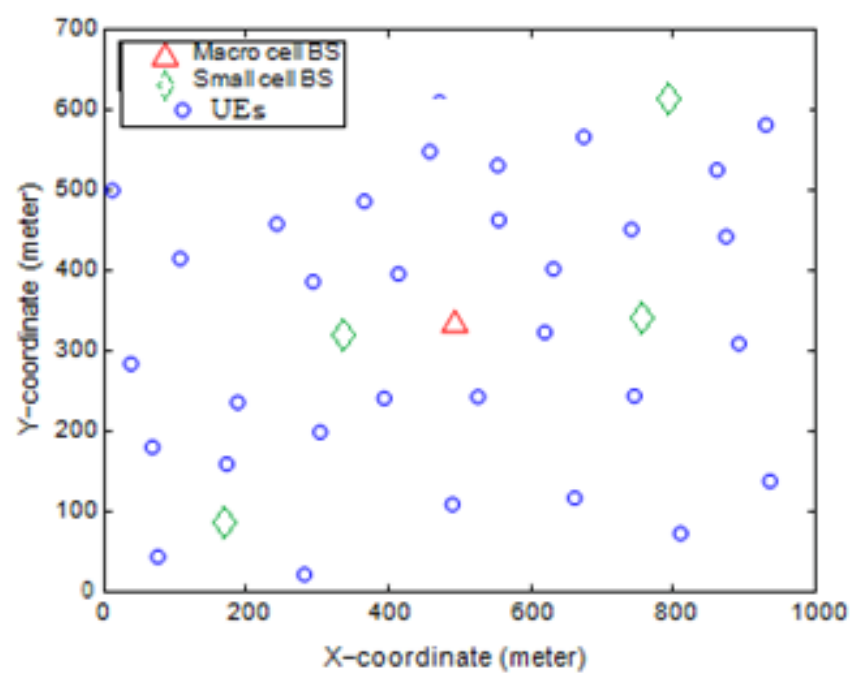

Figure 3 The geographic location (example)

The energy-efficient performance of the proposed approach we compare EE and power consumed with traditional energy and power consumed.

Our proposed work implemented is shown in Figure4 and Figure 5, which presents a comparative study analysis in terms of energy-efficient and power consumption, which compares the traditional $\mathrm{EE}$ and power consumed the use of the technique Beam-forming based on MVDR algorithm.

Figure 4 shows that energy consumption drained is less compared to the energy consumption after applying our approach proposed Beam-forming based on the MVDR algorithm.

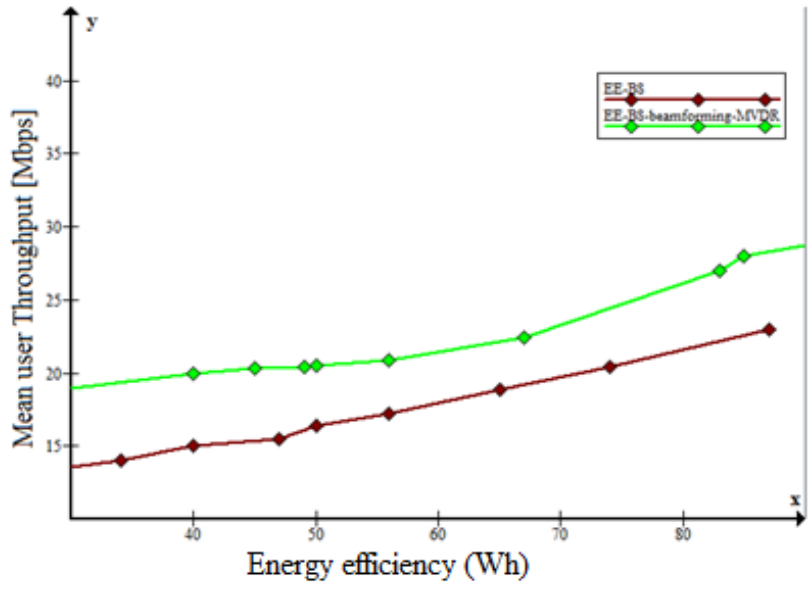

Figure 4 Energy efficiency of BS

Figure 5 shows that the power consumed by the basic is more comparatively to the Power consumed with the beamforming MVDR algorithm, in to order fined the optimum user of base station BS. 


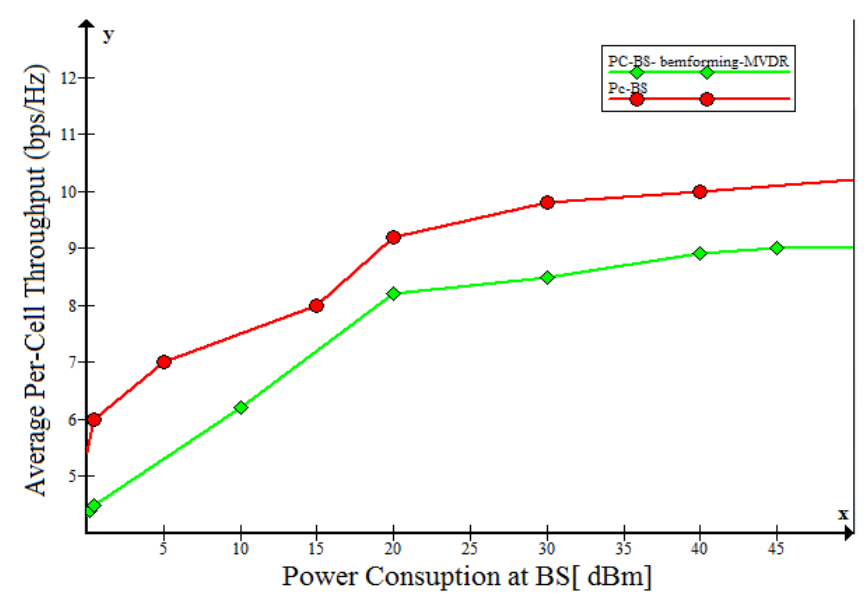

Figure 5 Power consumption of BS

\section{CONCLUSION}

The cellular networks have known a great development for responding to users 'needs. Several works have been investigated in this research field. However, it still suffers from the energy dissipation problem.

Different works proposed have designed to reduce the power and energy consumption of the wireless systems communication. In this work, we suggest to enhancing the energy efficiency using the adaptive beam-forming with MVDR algorithm. Our novel approach integrated permits the selecting the BS efficient and optimum users according to important adaptive, which minimize and reduced the energy consumed by BS. Simulation results have shown that our proposed is more efficient that the classical method.

\section{REFERENCES}

[1] Pedamallu, L. R. T., Singh, V. K., \& Gomes, A. P. F. (2016). Quantitative assessment of advanced energy efficiency retrofitting for hospitals in India. In ASME 2016 10th International Conference on Energy Sustainability, ES 2016.

[2] Al Haija, A. A., \& Tellambura, C. (2017). Small-Macro Cell Cooperation for HetNet Uplink Transmission: Spectral Efficiency and Reliability Analyses. In IEEE Journal on Selected Areas in Communications (Vol. 35, pp. 118-135).

[3] Abdulkafi, A. A., Kiong, T. S., Koh, J., Chieng, D., Ting, A., \& Ghaleb, A. M. (2012). Energy efficiency of LTE macro base station. In 2012 International Symposium on Telecommunication Technologies, ISTT 2012 (pp. 259-264).

[4] Isabona, J., \& Srivastava, V. M. (2017). Downlink Massive MIMO Systems: Achievable Sum Rates and Energy Efficiency Perspective for Future 5G Systems. Wireless Personal Communications, 96(2), 2779-2796. https://doi.org/10.1007/s11277-017-4324-y

[5] Murthy, C., \&Kavitha, C. (2012). A Survey of Green Base Stations in Cellular Networks. Ijcnwc.Org, 2(2), 232-236

[6] Monu Bhardwaj and Neelam. (2015). The Advantages and Disadvantages of Green Technology. Journal of Basic and Applied Engineering Research, 2(22), 1957-1960. Retrieved from http://libweb.surrey.ac.uk/library/skills/Introduction to Research and Managing Information Leicester/page_51.htm 
[7] Tavares, R., Mesquita, H., Penha, R., Abreu, P., \& Restivo, T. (2018). An instrumented glove for control audiovisual elements in performing arts. International Journal of Online Engineering, 14(2), 173-180. https://doi.org/10.3991/ijoe.v14i02.8247

[8] Fang, W., Yin, X., Y., Xiong, N., Guo, Q., \& Li, J. (2015). Optimal scheduling for data transmission between mobile devices and cloud. Information Sciences, 301, 169-180. https://doi.org/10.1016/j.ins.2014.12.059

[9] Hussain, S. S., Yaseen, S. M., \& Barman, K. (2016). An overview of massive mimo system in 5G. International Journal of Control Theory and Applications, 9(Specialissue1 1), 4957-4968.

[10] Tian, Y., Duan, H., \& Xue, Y. (2018). Nested Precoding in MU-MIMO Channels: The Superposition Transmission of Space-Time Coding and Spatial-Division Multiplexing. IEEE Transactions on Vehicular Technology, 67(6), 4867-4879. https://doi.org/10.1109/TVT.2018.2803149

[11] Anand, N., Lee, J., Lee, S. J., \& Knightly, E. W. (2015). Mode and user selection for multi-user MIMO WLANs without CSI. In Proceedings - IEEE INFOCOM (Vol. 26, pp. 451-459). https://doi.org/10.1109/INFOCOM.2015.7218411

[12] Wang, M., Samarasinghe, T., \& Evans, J. S. (2015). Optimizing user selection schemes in vector broadcast channels. IEEE Transactions on Communications, 63(2), 565-577. https://doi.org/10.1109/TCOMM.2015.2389816

[13] Wang, Z., Mei, L., Wang, X., \& Zhang, N. (2017). Bit error rate analysis of generalised frequency division multiplexing with weighted-type fractional Fourier transform precoding. IET Communications, 11(6). https://doi.org/10.1049/iet-com.2016.0772

[14] Biswas, A., \& Reisenfeld, S. (2017). New High Resolution Direction of Arrival Estimation using Compressive Sensing. 2017 IEEE 22nd International Workshop on Computer Aided Modeling and Design of Communication Links and Networks (CAMAD).

[15] Winterstein, A., \& Greda, L. A. (2018). An adaptive calibration and beamforming technique for a GEO satellite data relay. International Journal of Satellite Communications and Networking, 36(2), 207-219. https://doi.org/10.1002/sat.1213

[16] Wan, L., Sun, L., Wang, X., \& Bi, G. (2018). A range-Doppler-angle estimation method for passive bistatic radar. In 2018 International Conference on Signals and Systems, ICSigSys $2018 \quad$ - $\quad$ Proceedings $\quad$ (pp. https://doi.org/10.1109/ICSIGSYS.2018.8372669

[17] Hua, J., Lin, J., Zeng, L., \& Luo, Z. (2016). Minimum variance imaging based on correlation analysis of Lamb wave signals. Ultrasonics, 70, 107-122. https://doi.org/10.1016/j.ultras.2016.04.020

[18] Chaumette, E., Priot, B., Vincent, F., Pages, G., \& Dion, A. (2017). Minimum variance distortionless response estimators for linear discrete state-space models. IEEE Transactions on Automatic Control, 62(4), 2048-2055. https://doi.org/10.1109/TAC.2016.2594384

[19] Zorzi, M. M., Zanella, A., Member, S. S., Testolin, A., Grazia, M. D. F. De, Zorzi, M. M., Jha, R. K. (2016). A Traffic Load Balancing Framework for Software-Defined Radio Access Networks Powered by Hybrid Energy Sources. IEEE Communications Magazine, 18(2), 1-1. https://doi.org/10.1109/TNET.2015.2404576

[20] Haliloglu, O., Toker, C., Bulu, G., \& Yanikomeroglu, H. (2014). Energy efficient radio resource management in a coordinated multi-cell distributed antenna system. In IEEE International Symposium on Personal, Indoor and Mobile Radio Communications, PIMRC (Vol. 2014-June, pp. 872-876). https://doi.org/10.1109/PIMRC.2014.7136288 